\title{
POWER CONTROL IN WCDMA NETWORKS: A QUEUE-BASED APPROACH
}

\author{
Luigi Chisci * Romano Fantacci ** \\ Lorenzo Mucchi ${ }^{* *}$ Tommaso Pecorella ${ }^{* * *}$ \\ * Dipartimento di Sistemi e Informatica, Università di \\ Firenze, via Santa Marta 3, 50139 Firenze, Italy \\ e-mail: chisci@dsi.unifi.it \\ ** Dipartimento di Elettronica e Telecomunicazioni, \\ Università di Firenze, via Santa Marta 3, 50139 Firenze, \\ Italy \\ e-mail: $\{$ fantacci,mucchi\}@lenst.det.unifi.it \\ *** CNIT, Unità di Firenze, via Santa Marta 3, 50139 \\ Firenze, Italy \\ e-mail: pecos@lenst.det.unifi.it
}

\begin{abstract}
In modern cellular communication systems, power control plays a fundamental role for efficient resource utilization. In such systems, in fact, many users transmit over the same radio channel using the same frequency band and time slots so that the signal of an individual user becomes interference for the other users. Hence the transmission power levels need to be smartly manipulated so as to achieve an adequate quality of service for as many users as possible and, thus, an efficient network utilization. Conventional power control algorithms adopt the Signal-to-Interference-plus-Noise Ratio (SINR) as controlled variable and neglect the important effects of the manipulated control variables (transmission powers) and of the retransmission mechanism on the queueing dynamics. In this paper, we pursue a different queue-based approach which takes into account the queuing dynamics and adopts the queue size as controlled variable. In this framework, the power control problem is formulated as an optimal control problem with a criterion trading off high performance (low queue sizes) vs. low costs (low transmision powers). In particular, a novel queue-based power control algorithm with low on-line computational burden is proposed and its performance is evaluated via simulation experiments. The simulation results exhibit an improved behavior of the proposed queue-based algorithm compared to conventional SINR-based algorithms. Copyright $(2005$ IFAC
\end{abstract}

Keywords: Communication networks; communication control applications; power control; distributed control; predictive control. 


\section{INTRODUCTION}

Power control is a key issue for the efficient management of wireless communication networks as it attempts to mantain a satisfactory Quality of Service (QoS) for as many users as possible. In this paper, the focus will be on the uplink communication in a Wideband Code Division Multiple Access (WCDMA) system. In addition to multipath fading, shadowing and distance dependent propagation loss also cause signal attenuation in the communication channel. Since the spreading codes of different users cannot be orthogonal with all delays and with superimposed data modulation, the signals received at the base station interfere with each other (Pickholtz et al., 1991; Lee, 1991). If mobile stations transmit signals at a constant power level, the signalto-interference-plus-noise ratio (SINR) of the received signal at the base station of a distant mobile station would be too small for good quality communication due to nearby, strongly interfering mobile stations (not to mention the effect of thermal noise). This situation is called the near-far problem, and it appears in both single cell systems and cellular systems. The near-far problem can be compensated for by uplink power control. The transmission powers of mobile stations that belong to the same base station are adjusted so that the powers received at the base station have approximately the same minimum level necessary to accomplish good quality communication (Salmasi and Gilhousen, 1991). In this way, the interference from a mobile station to other mobile stations is minimized. Since the WCDMA system is interference limited, minimization of interference among all mobile stations contributes linearly to the system capacity (Gilhousen et al., 1991). Furthermore, uplink power control decreases power consumption of the mobile station (battery life is prolonged) and helps to overcome slow multipath fading (Salmasi, 1990).

In uplink power control, long-term attenuation is compensated for by an open loop method, in which a mobile station adjusts its transmission power according to its measurements. The open loop method is incapable of compensating for multipath fading occurring in the other link. Therefore, a closed loop, or feedback, method is required. In the closed loop method, the base station either

- commands, according to its measurements, a mobile station to adjust its transmission power (centralized power control CPC), or

- sends a metric describing the link quality, according to which the mobile station adjusts its transmission power (distributed power control - DPC) (Pichna and Wang, 1996).
In CPC (Zander, 1992; 3GPP, 1999), the base station can take into account the situations of multiple mobile stations when making a decision. However, CPC involves added infrastructure and introduces a feedback delay in the power control process, which degrades the system performance. For these reasons, CPC is inpractical and several DPC schemes have been proposed (Foschini and Miljanic, 1993; Yates, 1995; Gunnarsson et al., 1999; Alpcan et al., 2002; Alpcan and Başar, 2004; Gunnarsson and Gustafsson, 2003). In all the power control algorithms so far presented in the literature - see e.g. the survey papers of (Hanly and Tse, 1999) and (Gunnarsson and Gustafsson, 2003) - the primary control objective is expressed by the requirement that the SINR of each user does not exceed a pre-specified threshold determined by QoS specifications. In contrast to the conventional SINR-based approach, a novel queuebased approach will be pursued in the present paper. The idea of this approach is to express the QoS of each user in terms of its queue size (i.e. the number of packets waiting to be transmitted by the user) instead of SINR. To this end, the effect of the transmission power on the queue dynamics is modeled and power control can be cast into an optimal control problem with a criterion tradingoff the conflicting issues of high performance (low queues) and low costs (low transmission powers). In particular, a computationally efficient QueueBased Distributed Power Control (QBDPC) algorithm is proposed. The performance of QBDPC has been compared, via simulation experiments, with the performance of other SINR-based DPC algorithms having a similar on-line computational burden, showing that the queue-based approach is indeed promising.

The rest of the paper is organized as follows. Section 2 describes the uplink power control problem for a WCDMA system. Section 3 introduces the queue-based approach to power control and the QBDPC algorithm. Section 4 presents simulation results and finally section 5 ends the paper.

\section{POWER CONTROL IN WCDMA NETWORKS}

Let us consider a single-cell WCDMA system with $M$ Mobile Stations $\mathrm{MS}_{i}, i=1,2, \ldots, M$, sharing the same channel to the Base Station (BS). Let $g_{i}$ denote the channel gain between $\mathrm{MS}_{i}$ and BS and $u_{i}$ the transmission power from $\mathrm{MS}_{i}$ to BS. Then the achieved Signal to Interference plus Noise Ratio (SINR) for $\mathrm{MS}_{i}$ can be expressed as

$$
\gamma_{i}=\frac{L g_{i} u_{i}}{\sigma^{2}+\sum_{j \neq i} g_{j} u_{j}}
$$


where $\sigma^{2}>0$ is the noise power at the BS and $L>1$ is the spreading gain (chip rate to bit rate ratio) of the WCDMA system. The power control algorithms actually in use in WCDMA systems, as well as the ones so far proposed in the literature, adopt the SINR as controlled variable, i.e. adjust the transmission power $u_{i}$ so as to regulate the SINR $\gamma_{i}$ at a pre-specifed setpoint related to the desired QoS of user $\mathrm{MS}_{i}$. These algorithms will be referred to in this paper as SINR-based power control algorithms. For QoS management, however, it seems more appropriate to control the queue size in each MS rather than the SINR. In fact, the QoS perceived by each user is expressed in terms of the service delay and of the packet loss rate which, in turn, are directly related to the size of the user's queue, i.e. the number of packets waiting to be transmitted by the user. Hence, it might be advisable to either reduce or increase the transmission power depending on the current queue state rather than on the current SINR.

For this reason a different queue-based approach to distributed power control will be described in the next section.

\section{QUEUE-BASED APPROACH TO POWER CONTROL}

Each user $\mathrm{MS}_{i}$ is modelled as a queue with state equation

$$
x_{i}(t+1)=x_{i}(t)+w_{i}(t)-v_{i}(t)
$$

where $x_{i}(t), w_{i}(t), v_{i}(t)$ denote respectively the queue size, input flow, output flow (measured in packets) at sample time $t$. In turn, the output flow $v_{i}(t)$ is modeled by

$$
v_{i}(t)=R\left(1-P E R_{i}(t)\right)
$$

where $R$ is the fixed channel transmission capacity (packets transmitted per sampling period) and $P E R_{i}(t)$ is the packet error rate of $\mathrm{MS}_{i}$ at sample time $t$. Finally it is assumed that the PER is a known decreasing function of the SINR, i.e.

$$
P E R_{i}(t)=G\left(\gamma_{i}(t)\right) .
$$

Notice that, combining (3) and (4), the output flow is related to the SINR by

$$
v_{i}(t)=F\left(\gamma_{i}(t)\right)=F_{i}(u(t))
$$

where: $F(\gamma) \triangleq R(1-G(\gamma))$ is an increasing function of $\gamma ; u=\left[u_{1}, u_{2}, \ldots, u_{M}\right]$ is the vector of all transmission powers and $F_{i}(u) \triangleq$ $F\left(\left(\sigma^{2}+\sum_{j \neq i} g_{j} u_{j}\right)^{-1} L g_{i} u_{i}\right)$. In a centralized power control scheme, the objective would be to choose, at each sampling time $t$, the whole control vector $u(t)$ based on the knowledge of the whole state vector $x(t)=\left[x_{1}(t), x_{2}(t), \ldots, x_{M}(t)\right]$ so as to possibly optimize some global network utility. In particular, a predictive control approach could be pursued by defining a suitable finite-horizon cost $J(x(t) ; u(t), u(t+1), \ldots, u(t+H-1))$ which trades off QoS (low queue sizes) vs. battery usage (low powers), and by designing the power control $u(t)$, at time $t$, as the first element of the optimal control sequence minimizing such a cost according to the so called receding-horizon strategy. However, centralized power control is not viable in practice and a decentralized (distributed) control scheme, wherein each user $\mathrm{MS}_{i}$ selects its own transmission power $u_{i}$ based on local information only, must be adopted. In this context it is assumed that user $\mathrm{MS}_{i}$, besides the knowledge of the local state $x_{i}$ and input flow $w_{i}$, has also measurements (or estimates) of the gain $g_{i}$ and of the total interference-plus-noise power at the BS (denominator of the SINR)

$$
\sigma_{i}^{2}=\sigma^{2}+\sum_{j \neq i} g_{j} u_{j}
$$

It is further assumed that such quantities $g_{i}(t)$ and $\sigma_{i}^{2}(t)$ are slowly varying with time $t$ and that, for the purpose of control design, the available estimates are exact. Exploiting (1), (5) and (6), the transmission power $u_{i}(t)$ turns out to be related to the output flow $v_{i}(t)$ by

$$
u_{i}(t)=\frac{\sigma_{i}^{2}(t)}{L g_{i}(t)} F^{-1}\left(v_{i}(t)\right)
$$

The goal of distributed power control is, therefore, to design a power control law $u_{i}=u_{i}\left(x_{i}, w_{i}, g_{i}, \sigma_{i}^{2}\right)$ in each mobile station $\mathrm{MS}_{i}$ so as to optimize a local performance index. To this end, the following cost is introduced for $\mathrm{MS}_{i}$

$$
J_{i}\left(x_{i}(t), u_{i}(t)\right)=x_{i}^{2}(t+1)+r_{i} v_{i}^{2}(t)
$$

where $r_{i}>0$. Notice that in (8) a control horizon $H=1$ has been chosen and a quadratic penalization $v_{i}^{2}$, instead of $u_{i}^{2}$, has been considered to avoid nonlinearity of $(2)$ w.r.t. $u_{i}$. It will be seen hereafter that these choices greatly simplify the underlying optimal control problem and also provide a resulting control law with low on-line computational burden. The Queue-Based Distributed Power Control ( $Q B D P C$ ) problem is therefore formulated as follows:

$$
\begin{aligned}
& \text { for } i=1,2, \ldots, M: \\
& u_{i}(t)=\arg \min _{u_{i}(t)} x_{i}^{2}(t+1)+r_{i} v_{i}^{2}(t)
\end{aligned}
$$

subject to 


$$
\left\{\begin{aligned}
x_{i}(t+1) & =x_{i}(t)+w_{i}(t)-v_{i}(t) \\
v_{i}(t) & =F\left(\frac{L g_{i}(t)}{\sigma_{i}^{2}(t)} u_{i}(t)\right) \\
\underline{u} & \leq u_{i}(t) \leq \bar{u}
\end{aligned}\right.
$$

where $\underline{u}$ and $\bar{u}$ represent the lower and, respectively, upper limitation on the transmission power of a MS.

Theorem 1 - The solution to the above QBDPC problem (9)-(10) is given by the following QBDPC control law

$$
\begin{aligned}
\text { For } i & =1,2, \ldots, M \\
v_{i}^{*}(t) & =\frac{1}{1+r_{i}}\left(x_{i}(t)+w_{i}(t)\right) \\
\gamma_{i}^{*}(t) & =F^{-1}\left(v_{i}^{*}(t)\right) \\
u_{i}^{*}(t) & =\frac{\sigma_{i}^{2}(t)}{L g_{i}(t)} \gamma_{i}^{*}(t) \\
u_{i}(t) & =\operatorname{sat}\left(u_{i}^{*}(t)\right)
\end{aligned}
$$

where

$$
\operatorname{sat}(u) \triangleq\left\{\begin{array}{l}
\underline{u}, u<\underline{u} \\
\bar{u}, \underline{u} \leq \bar{u} \leq \bar{u} \\
\bar{u}, u>\bar{u}
\end{array}\right.
$$

Proof - Since the map between $u_{i}$ and $v_{i}$ is monotonic increasing, the bounds on $u_{i}$ induce corresponding bounds on $v_{i}$ (and viceversa). Hence the problem (9)-(10) can be restated in terms of $v_{i}$ as

$$
\min _{v_{i}(t)}\left(1+r_{i}\right) v_{i}^{2}(t)-2\left(x_{i}(t)+w_{i}(t)\right) v_{i}(t)
$$

subject to

$$
\underline{v}_{i}(t) \leq v_{i}(t) \leq \bar{v}_{i}(t)
$$

where $\underline{v}_{i}(t) \triangleq F\left(\frac{L g_{i}(t)}{\sigma_{i}^{2}(t)} \underline{u}\right)$ and $\bar{v}_{i}(t) \triangleq F\left(\frac{L g_{i}(t)}{\sigma_{i}^{2}(t)} \bar{u}\right)$. Due to the parabolic form of the cost (12) and the interval form of the constraints (13), the constrained solution of the scalar optimization problem (12)-(13) is trivially obtained as the saturated unconstrained solution of (12), i.e.

$$
v_{i}(t)=\operatorname{sat}\left(v_{i}^{*}(t)\right)
$$

where $v_{i}^{*}(t)$ is given in (11) and the saturation bounds are $\underline{v}_{i}(t)$ and $\bar{v}_{i}(t)$. Then the optimal transmission power $u_{i}(t)$ is obtained by the inverse transformation of (7), i.e.

$$
u_{i}(t)=\frac{\sigma_{i}^{2}(t)}{L g_{i}(t)} F^{-1}\left(\operatorname{sat}\left(v_{i}^{*}(t)\right)\right)
$$

Due to the monotonic increase of $F^{-1}$, it is possible to interchange the operators $F^{-1}$ and sat in (15) and thus obtain

$$
u_{i}(t)=\operatorname{sat}\left(\frac{\sigma_{i}^{2}(t)}{L g_{i}(t)} F^{-1}\left(\frac{1}{1+r_{i}}\left(x_{i}(t)+w_{i}(t)\right)\right)\right)
$$

which is just the expression provided by (11) in explicit form.

Please notice that the proposed QBDPC control law involves only few algebraic calculations and is therefore amenable to fast on-line implementation in a WCDMA system, without requiring excessive processing capabilities to the mobile stations. Performance can be tuned by each mobile station $\mathrm{MS}_{i}$ by properly selecting, possibly in a time-varying fashion, the parameter $r_{i}>0$. A possible way is to choose $r_{i}$ based on some measured indicator of QoS, so that $r_{i}$ is taken larger (smaller) whenever the measured QoS is higher (lower). Performance issues will be investigated in the next section by means of simulation experiments.

\section{SIMULATION RESULTS}

The QBDPC algorithm described in the previous section is compared with the following two SINRbased algorithms:

- the Up/Down Centralized Power Control algorithm which has been standardized by 3GPP (1999) to be used in WCDMA;

- the DPC algorithm of Alpcan et al. (2002) which is based on game theory and will be referred to hereafter as Game-Theoretic DPC $(G T D P C)$.

The UDCPC algorithm is run at the BS and assumes centralized information on all $\gamma_{i}(t)$ for $i=1,2, \ldots, M$. More precisely, UDCPC operates as follows

$$
u_{i}(t)=u_{i}(t-1)+\operatorname{sign}\left(\gamma_{i}^{*}-\gamma_{i}(t)\right) \delta u
$$

where $\delta u$ is a fixed power step while $\gamma_{i}^{*}$ and $\gamma_{i}(t)$ are the target and, respectively, estimated SINR of $\mathrm{MS}_{i}$. This power update (fixed step increase/decrease) is typically performed at a rate of $1.5 \mathrm{KHz}$.

The GTDPC algorithm is, conversely, a distributed algorithm run in each $\mathrm{MS}_{i}$. It is based on minimization in each $\mathrm{MS}_{i}$ of a local cost function

$$
J_{i}\left(u_{i}, \gamma_{i}\right)=\alpha_{i} u_{i}-\beta_{i} \log \left(1+\gamma_{i}\right)
$$

where the first represents a penalty proportional to the used transmission power and the second term an utility proportional to the channel capacity. The resulting GTDPC control law is given by

$$
u_{i}(t)=\max \left(0, \frac{\beta_{i}}{\alpha_{i}}-\frac{\sigma_{i}^{2}(t)}{L g_{i}(t)}\right) .
$$

In order to comparatively evaluate the performance of the QBDPC, UDCPC and GTDPC algorithms, a WCDMA communication system has been simulated. The simulations have been run under the following hypotheses: 
Table 1. Types of traffic flow per user.

\begin{tabular}{cccc} 
Type of traffic & Rate & L & Coding \\
\hline Voice & $60 \mathrm{kbps}$ & 64 & $1 / 2$ convolutional \\
Data (interactive) & $480 \mathrm{kbps}$ & 8 & $1 / 3$ turbocode
\end{tabular}

Table 2. Simulation parameters.

$\begin{array}{ll}\text { Minimum time unit } & 10^{-7} \mathrm{sec} \\ \text { Packet time } & 0.667 \mathrm{msec} \\ \text { Acknowledge time of one packet } & 10^{-5} \mathrm{sec} \\ \text { Multipath fading variance (rural) } & -3.6 \mathrm{~dB} \\ \text { Multipath fading variance (urban) } & -5.7 \mathrm{~dB} \\ \text { Start up MS transmission power } & 10 \mathrm{~mW} \\ \text { Minimum transmission power } & 1 \mathrm{~mW} \\ \text { Maximum transmission power (voice) } & 125 \mathrm{~mW} \\ \text { Maximum transmission power (data) } & 250 \mathrm{~mW} \\ \text { Frequency of QBDPC } & 200 \mathrm{~Hz} \\ \text { Frequency of UDCPC } & 1500 \mathrm{~Hz} \\ \text { Frequency of GTDPC } & 200 \mathrm{~Hz} \\ \text { Parameter } r_{i} \text { of QBDPC } & \log _{2}\left(1+\gamma_{i}\right)\end{array}$

- single cell controlled by one base station (BS);

- up link;

- mobile stations (MS) have different distances to the BS (near-far);

- each user (MS) has an asynchronous packet traffic flow to the BS with ARQ Stop and Wait protocol;

- each MS can have two different types of traffic: voice and data (details in table 1);

- the background noise power is fixed at $10 \mathrm{~dB}$ lower than the signal;

- perfect SINR estimation is supposed;

- the PER target for the voice service is $10 \%$, while data has $0.1 \%$;

- the relation between the PER and the SINR (4) for coded transmissions can be extracted from (Souto et al., 2003).

A multipath fading channel has been modeled (3GPP, 1999). The channel has 4 taps whose amplitudes and phases are Rayleigh and uniform distributed, respectively. Moreover, two possible user mobilities (Doppler frequency, $f_{d}$ ) have been considered: pedestrian $\left(f_{d}=3 \mathrm{~Hz}\right)$ and vehicular $\left(f_{d}=120 \mathrm{~Hz}\right)$.

The following simulation experiment, of duration equal to $6500 \mathrm{~s}$, has beeen performed: a first group of 4 users (mobile stations) is initially present in the cell; then a second group of 3 users enters the cell at time $t_{1}=2500 \mathrm{~s}$; next, the users of the first group leave the cell at time $t_{2}=4500 \mathrm{~s}$. All the simulation parameters are reported in table 2 .

In figs. 1 and 2 the behavior of the number of queued packets for the three algorithms is shown. It can be seen that UDCPC, QBDPC and GTDPC have comparable queue sizes. On the other hand, if the comparison is switched to the average transmission power (see figs. 3 and 4), it can be noticed that the QBDPC reaches the same queue sizes with lower transmission powers compared

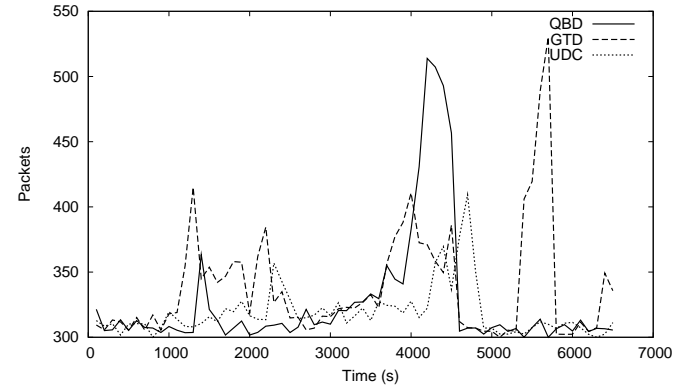

Fig. 1. Voice queue sizes for QBDPC, GTDPC and UDCPC.

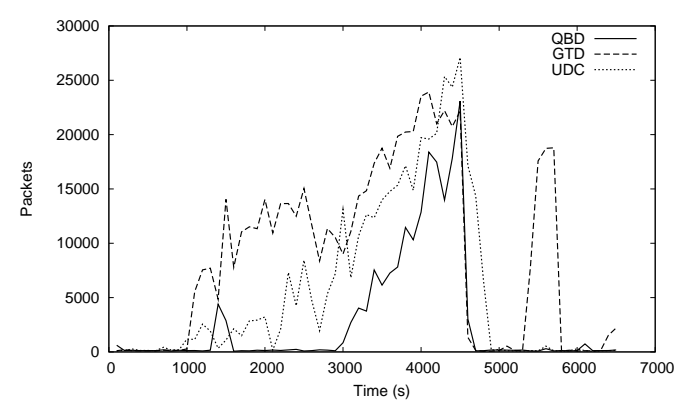

Fig. 2. Data queue sizes for QBDPC, GTDPC and UDCPC.

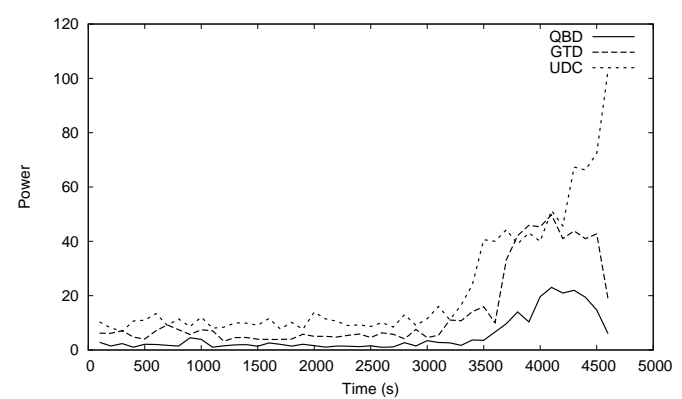

Fig. 3. Average transmission powers for the voice packets of QBDPC, GTDPC and UDCPC.

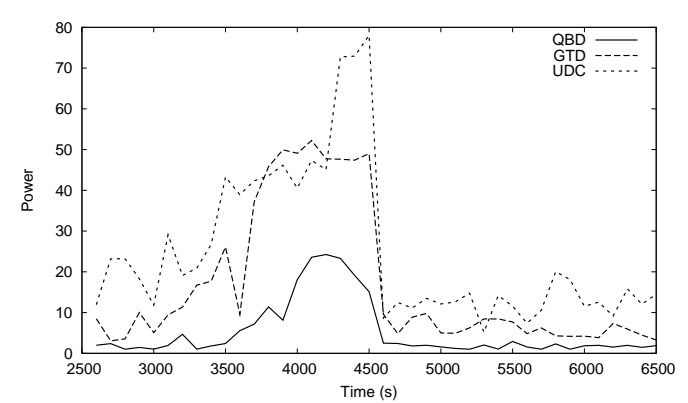

Fig. 4. Average transmission powers for the data packets of the QBDPC, GTDPC and UDCPC.

to the GTDPC and UDCPC algorithms. Finally, from fig. 5 it is possible to appreciate that, for a given SINR, the QBDPC algorithm is able to efficiently save power compared to GTDPC and even more to UDCPC. These conclusions can be drawn by looking at the voice or data information sources, indifferently. 

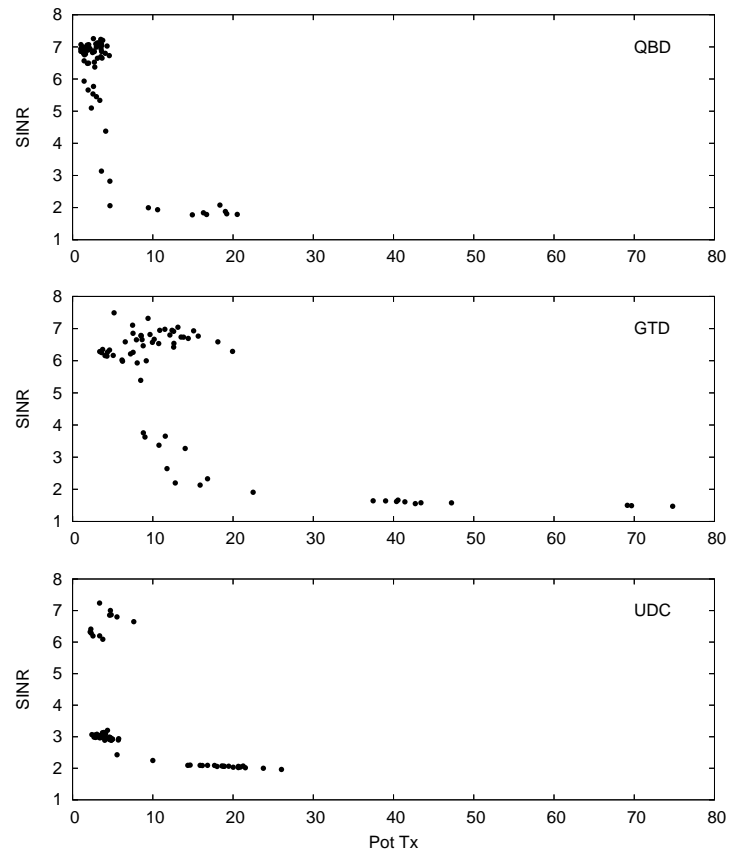

Fig. 5. Average transmission power versus SINR for the data packets of QBDPC, GTDPC and UDCPC.

\section{CONCLUSIONS}

The paper has presented a novel queue-based approach to power control in wireless WCDMA communication networks. This approach relies on modelling how the transmission powers affect the queueing dynamics and formulates power control as an optimal control problem. Following this "queue" paradigm, a novel computationally efficient queue-based distributed power control (QB$D P C$ ) algorithm has been devised. Simulation results demonstrate the effectiveness of QBDPC compared to existing SINR-based algorithms. In particular, it has been seen that QBDPC exhibits comparable performance with significant power savings with respect to the up/down centralized algorithm currently used in WCDMA networks as well as with respect to a recently proposed distributed algorithm based on game theory. Future work will be devoted to the theoretical analysis of the stability and performance properties of the proposed algorithm.

\section{REFERENCES}

3GPP (1999). Technical specification group radio access network. Technical report. Standard document series 3G TS 25 Release 1999.

Alpcan, T. and T. Başar (2004). A hybrid systems model for power control in multicell wireless data networks. Performance Evaluation 47, 477-495.

Alpcan, T., T. Başar, R. Srikant and E. Altman (2002). CDMA uplink power control as a noncooperative game. Wireless Networks, The Journal of Mobile Communication, Computation and Information 8, 659-670.

Foschini, G.J. and Z. Miljanic (1993). A simple distributed power control algorithm and its convergence. IEEE Transactions on Vehicular Technology 42, 641-646.

Gilhousen, S., I.M. Jacobs, R. Padovani, A.J. Viterbi, L.A. Weaver and C.E. Wheatley III (1991). On the capacity of a cellular CDMA system. IEEE Transactions on Vehicular Technology 40(2), 303-312.

Gunnarsson, F. and F. Gustafsson (2003). Control theory aspects of power control in UMTS. Control Engineering Practice 11, 1113-1125.

Gunnarsson, F., F. Gustafsson and J. Blom (1999). Pole placement design of power control algorithms. In: Proc. of the IEEE 49th Vehicular Technology Conference. Houston, USA. pp. 2149-2153.

Hanly, S.V. and D.N. Tse (1999). Power control and capacity of spread spectrum wireless networks. Automatica 35, 1987-2012.

Lee, W.C.Y. (1991). Overview of cellular CDMA. IEEE Transactions on Vehicular Technology 40(2), 291-302.

Pichna, R. and Q. Wang (1996). The Mobile Communications Handbook. CRC Press, New York.

Pickholtz, R.L., L.B Milstein and D.L. Schilling (1991). Spread spectrum for mobile communications. IEEE Transactions on Vehicular Technology 40(2), 313-322.

Salmasi, A. (1990). An overview of code division multiple access CDMA applied to the design of personal communications networks. In: Proc. Second Rutgers University WINLAB Workshop on the Third Generation Wireless Information Networks. East Brunswick, USA. pp. $277-298$.

Salmasi, A. and S. Gilhousen (1991). On the system design aspects of code division multiple access (CDMA) applied to digital cellular and personal communications networks. In: Proc. IEEE Vehicular Technology Conference. St. Louis, USA. pp. 57-62.

Souto, N., J.C. Silva, A. Correia, F. Cercas and A. Rodrigues (2003). UMTS AWGN simulation results for uplink, downlink and HSDPA transmissions. In: Proc. IEEE 58th Vehicular Technology Conference. pp. 128-132.

Yates, R.D. (1995). A framework for uplink power control in cellular radio systems. IEEE Journal on Selected Areas in Communications 13, 1341-1347.

Zander, J. (1992). Performance of optimum transmitter power control in cellular radio systems. IEEE Transactions on Vehicular Technology 41, 57-62. 